\title{
STK31 upregulation is associated with chromatin remodeling in gastric cancer and induction of tumorigenicity in a xenograft mouse model
}

\author{
DONG HYUCK BAE ${ }^{1,2}$, HEE-JIN KIM ${ }^{1}$, BYOUNG-HA YOON ${ }^{3}$, JONG-LYUL PARK ${ }^{1}$, MIRANG KIM ${ }^{2,3}$, \\ SEON-KYU KIM ${ }^{3,4}$, SEON-YOUNG KIM ${ }^{2,3}$, SANG-IL LEE ${ }^{5}$, KYU-SANG SONG ${ }^{6}$ and YONG SUNG KIM ${ }^{1,2}$ \\ ${ }^{1}$ Genome Editing Research Center, Korea Research Institute of Bioscience and Biotechnology, Daejeon 34141; \\ ${ }^{2}$ Department of Functional Genomics, Korea University of Science and Technology, Daejeon 34113; \\ ${ }^{3}$ Personalized Genomic Medicine Research Center, Korea Research Institute of Bioscience and Biotechnology, Daejeon 34141; \\ ${ }^{4}$ Department of Bioinformatics, Korea University of Science and Technology, Daejeon 34113; \\ Departments of ${ }^{5}$ Surgery and ${ }^{6}$ Pathology, College of Medicine, Chungnam National University, \\ Daejeon 35015, Republic of Korea
}

Received October 5, 2020; Accepted January 19, 2021

DOI: $10.3892 / o r .2021 .7993$

\begin{abstract}
Pathological changes in the epigenetic landscape of chromatin are hallmarks of cancer. Our previous study showed that global methylation of promoters may increase or decrease during the transition from gastric mucosa to intestinal metaplasia (IM) to gastric cancer (GC). Here, $\mathrm{CpG}$ hypomethylation of the serine/threonine kinase STK31 promoter in IM and GC was detected in a reduced representation bisulfite sequencing database. STK31 hypomethylation, which resulted in its upregulation in 120 cases of primary GC, was confirmed. Using public genome-wide histone modification data, upregulation of STK31 promoter activity was detected in primary GC but not in normal mucosae, suggesting that STK31 may be repressed in gastric mucosa but activated in $\mathrm{GC}$ as a consequence of hypomethylation-associated chromatin remodeling. STK31 knockdown suppressed the proliferation, colony formation and migration activities of GC cells in vitro, whereas stable overexpression of $S T K 31$ promoted the proliferation, colony formation, and migration activities of GC cells in vitro and tumorigenesis in nude mice. Patients with GC in which STK3I was upregulated exhibited significantly shorter survival times in a combined cohort. Thus, activation of STK31 by chromatin remodeling may be associated with gastric carcinogenesis and also may help predict GC prognosis.
\end{abstract}

Correspondence to: Dr Yong Sung Kim, Genome Editing Research Center, Korea Research Institute of Bioscience and Biotechnology, 125 Gwahak-ro, Yuseong-gu, Daejeon 34141, Republic of Korea E-mail: yongsung@kribb.re.kr

Key words: serine/threonine kinase 31, gastric cancer, DNA hypomethylation, chromatin remodeling, prognosis

\section{Introduction}

Gastric cancer (GC) is one of the most common types of malignancy, more than one million cases are diagnosed each year worldwide and survival rate decreases as cancer progresses $(1,2)$. Many patients with GC are diagnosed at an advanced stage because GC is initially asymptomatic and biomarkers are lacking (2). Therefore, the identification of biomarkers for early-stage detection and prediction of prognosis may improve the efficacy of GC treatment strategies (3).

Gastric carcinogenesis is a multistep process that arises from superficial gastritis and progresses to chronic atrophic gastritis, intestinal metaplasia (IM), dysplasia and carcinoma (4). Similar to other types of cancer, gastric carcinogenesis exhibits a multifactorial etiology involving environmental, genetic and epigenetic components. Among epigenetic alterations, there has been interest in hypermethylation/repression of tumor-suppressor genes (5). Moreover, it is hypothesized that DNA hypomethylation promotes cancer development via activation of proto-oncogenes (6), although examples of this are lacking. However, advances in global methylation profiling suggest that aberrant promoter hypomethylation is a frequent event in hematological malignancies, such as chronic lymphocytic leukemia (7).

Our previous study (8) used genome-wide reduced representation bisulfite sequencing (RRBS) and methyl-CpG binding domain sequencing of GC specimens and found that gastrointestinal hormone receptor genes in a neuroactive ligand-receptor interaction pathway are predominantly hypermethylated in GC. STK31 serves a role in spermatogenesis in human testes (9-12). STK31 expression is normally restricted to the testis, yet it is frequently overexpressed not only in colorectal and esophageal cancer but also in GC; it is therefore referred to as a cancer/testis antigen gene (13), which include $M A G E-A 1$ and MAGE-A3. Moreover, a previous study (14) suggested that aberrant expression of STK31 contributes to tumorigenicity in somatic cancer cells, and thus STK31 
and STK31 may be potential therapeutic targets in human somatic cancer. In colorectal cancer, for example, STK31 expression can be reactivated by treating diseased tissue with 5-aza-2-deoxycytidine (5-aza-dC) (13). The kinase domain of STK31 regulates tumorigenicity via control of differentiation state, suggesting that STK31 may be regulated by an epigenetic mechanism (15). However, the role of STK31 in GC and the mechanism by which STK31 transcription is controlled in GC is not clear.

The aim of the present study was to determine whether the regulation of STK31 expression is associated with chromatin remodeling, including DNA methylation and histone modification at the promoter region, and to elucidate the role of STK3 in gastric carcinogenesis. In order to reveal the epigenetic alteration in IM and/or GC, methylome data of the RRBS and 450K HumanMethylation BeadChip data and publicly available genome-wide histone modification data were used. In order to identify the role of STK31 in GC cells, in vitro experiments, such as proliferation, colony forming and migration assays in stable GC cells, and in vivo experiments with xenograft mice were performed. Moreover, patients for which STK31 was upregulated were investigated for survival times in a combined cohort. These results may provide insight into the role of for STK31 in the development of GC and as a potential therapeutic and prognostic target for patients with GC.

\section{Materials and methods}

Cell lines and tissue samples. A total of 16 GC cell lines, namely SNU001, SNU005, SNU016, SNU216, SNU484, SNU520, SNU601, SNU620, SNU638, SNU668, SNU719, AGS, KATOIII, MKN01, MKN45 and MKN74, were obtained from the Korean Cell Line Bank (cellbank.snu.ac.kr/main/index. html) and cultured in RPMI-1640 medium (Welgene, Inc.) supplemented with $10 \%$ fetal bovine serum and $1 \%$ antibiotic-antimycotic solution (Invitrogen; Thermo Fisher Scientific, Inc.) at $37^{\circ} \mathrm{C}$ in a humidified $5 \% \mathrm{CO}_{2}$ incubator. All experiments were performed with mycoplasma-free cells and all GC cell lines were authenticated using short tandem repeat profiling within the past 3 years. A total of 145 frozen tumor and paired adjacent non-tumor tissue samples were obtained from the Chungnam National University Hospital (CNUH; Daejeon, South Korea), a member of the Korea Biobank Network. The samples included 31 stage I, 30 stage II, 46 stage III and 38 stage IV tumors from 50 females and 95 males, aged 23-83 years (average age, 59 years). All samples were obtained with informed consent and their use was approved by the Internal Review Board of CNUH (approval no. CNUH 2018-01-056).

Bisulfite sequencing. Bisulfite sequencing was performed on two different regions proximal to the STK31 promoter. Two sets of PCR primer were designed using Methprimer (urogene. org/cgi-bin/methprimer/methprimer.cgi): Region 1, forward, 5'-TCTAGAAAATGCAAACTAATATATGGTGAC-3' and reverse, 5'-GAAAGGAACTGGCCCTAGACCCCAC-3' to yield a 581-bp product containing $9 \mathrm{CpG}$ sites and Region 2, forward, 5'-GAAAGGAATTGGTTTTAGATTTTAT-3' and reverse, 5'-TCTAACACCCCTCTAAAATAAC-3' to yield a 468-bp product containing $36 \mathrm{CpG}$ sites. Genomic DNA $(2 \mu \mathrm{g})$ from GC cells or tissue was modified by sodium bisulfite using an EZ DNA methylation kit (Zymo Research Corp.). Bisulfite-modified DNA $(1 \mu \mathrm{l})$ was amplified in a $20 \mu \mathrm{l}$ volume using 2X Dye Mix polymerase (Enzynomics Co., Ltd.) and the aforementioned primers. Samples were heated to $95^{\circ} \mathrm{C}$ for $10 \mathrm{~min}$ and amplified for 40 cycles of $95^{\circ} \mathrm{C}$ for $45 \mathrm{sec}, 60^{\circ} \mathrm{C}$ for $45 \mathrm{sec}$ and $72^{\circ} \mathrm{C}$ for $60 \mathrm{sec}$, then incubated at $72^{\circ} \mathrm{C}$ for $10 \mathrm{~min}$ and cooled to $4^{\circ} \mathrm{C}$. The PCR products were visualized on a $1 \%$ agarose gel by ethidium bromide staining, purified from the gel using a Qiagen Gel Extraction kit (Qiagen, Inc.) and cloned using the pGEM-T Easy Vector (Promega Corporation). A total of ten clones were randomly chosen for sequencing. Complete bisulfite conversion was assured when $<0.01 \%$ of cytosines in non-CG dinucleotides in the final sequence had not converted.

Reverse transcription-quantitative $(R T-q) P C R$. Total RNA was extracted from GC cells or tissue using the RNeasy plus mini kit (Qiagen), treated with DNase I (Promega Corporation), and reverse-transcribed with Superscript II reverse transcriptase (Invitrogen; Thermo Fisher Scientific, Inc.). RT-qPCR for STK31 was performed with a CFX96 Real-Time PCR Detection system (Bio-Rad Laboratories, Inc.) using the following thermocycling conditions: $94^{\circ} \mathrm{C}$ for $5 \mathrm{~min}$, followed by 35 cycles of $94^{\circ} \mathrm{C}$ for $30 \mathrm{sec}, 64^{\circ} \mathrm{C}$ for $30 \mathrm{sec}$ and $72^{\circ} \mathrm{C}$ for $30 \mathrm{sec}$, with a final step of $72^{\circ} \mathrm{C}$ for $7 \mathrm{~min}$. $\beta$-actin served as the PCR control. The PCR products were analyzed on $1.5 \%$ agarose gels stained with ethidium bromide. The primer sequences for RT-qPCR were as follows: STK31 forward, 5'-AACCTGCTTCTCCAG GTTCA-3' and reverse, 5'-ATTGCTCCTTTGGCATCAAG-3' to yield a 228-bp product and $\beta$-actin forward, 5'-CAAGAG ATGGCCACGGCTGCT-3' and reverse, 5'-TCCTTCTGC ATCCTGTCGGCA-3' to yield a 275-bp product. RT-qPCR for STK31 was performed using a C1000 Thermal Cycler (Bio-Rad Laboratories, Inc.). cDNA (100 ng) was amplified as aforementioned for 45 cycles with $2 X$ SYBR-Green Supermix (Bio-Rad Laboratories, Inc.). $\beta$-actin was used as a control. The relative amount of target mRNA was quantified using comparative threshold cycle (Cq) method (16).

Pyrosequencing. Two CpG sites, namely CpG\#23 and \#24, in Region 2 were selected for quantification of the extent of methylation. Bisulfite-modified DNA (100 ng) was amplified by $\mathrm{PCR}$ in a $20 \mu \mathrm{l}$ reaction using $2 \mathrm{X}$ Dye Mix polymerase (Enzynomics Co., Ltd.) to yield a 221-bp product using the following primers: Forward, 5'-TGTTTGGGGGTAGGTAGT AGTTAG-3 and reverse, 5'-CCCTAAACCCACATACTAAAC TTTC-3'. PCR was performed using an initial melting step of $94^{\circ} \mathrm{C}$ for $5 \mathrm{~min}$, followed by 40 cycles of $94^{\circ} \mathrm{C}$ for $30 \mathrm{sec}, 59^{\circ} \mathrm{C}$ for $30 \mathrm{sec}$ and $72^{\circ} \mathrm{C}$ for $30 \mathrm{sec}$, with a final incubation at $72^{\circ} \mathrm{C}$ for $7 \mathrm{~min}$. Pyrosequencing was performed as previously described (17) using a sequencing primer (5'-AGGAGTAGT GTGGGGTTT-3') and PSQ HS 96A system (Biotage AB).

Treatment with 5-aza-dC and trichostatin A (TSA). SNU638, SNU719 and MKN74 cells were maintained as aforementioned. Cells were seeded in 100-mm dishes at a density of $1 \times 10^{6}$ cells per dish, then treated with $10 \mu \mathrm{M}$ DNA methylation inhibitor 5-aza-dC (Sigma-Aldrich; Merck KGaA) every $24 \mathrm{~h}$ for 3 days and harvested. Another group of these cells at the same cell density was treated with $0.5 \mu \mathrm{M}$ histone deacetylase inhibitor TSA (Sigma-Aldrich; Merck KGaA) for 3 days and harvested. In 
order to test the combined effect of 5-aza-dC and TSA, cells were treated with $10 \mu \mathrm{M} 5$-aza-dC every $24 \mathrm{~h}$ for 3 days and then with $0.5 \mu \mathrm{M}$ TSA for 1 day. After 2-5 days, cells were washed with phosphate-buffered saline, and total RNA was extracted using an RNeasy Mini kit (Qiagen, Inc.). All experiments were performed at $37^{\circ} \mathrm{C}$. RT-qPCR for $S T K 31$ was performed as aforementioned. A total of three independent experiments was performed.

Establishment of stable cell lines. STK31-knockdown (KD) cells were established using TRCN0000368917 and TRCN0000003276 (STK31_sh\#1 and STK31_sh\#4, respectively; Sigma-Aldrich; Merck KGaA) targeting STK31 mRNA; pLKO.1-puro (Sigma-Aldrich; Merck KGaA) was used as a control. For lentivirus construction, 293T cells were obtained from Koram Biotech Corp. and co-transfected with $2 \mu \mathrm{g}$ MISSION Lentiviral Packaging Mix and $2 \mu \mathrm{g}$ control or STK31 short hairpin (sh) RNA using a 2nd Generation lentiviral system and Lipofectamine ${ }^{\circledR} 2000$ (Invitrogen; Thermo Fisher Scientific, Inc.). In order to establish STK31-expressing cell lines, $2 \mu \mathrm{g}$ full-length STK31 cDNA was cloned into vector pCDH-CMV-MCS-EF1-Puro (System Biosciences, LLC). For lentivirus construction, 293T cells were co-transfected using a 2nd generation lentiviral system with $2 \mu \mathrm{g}$ MISSION Lentiviral Packaging Mix plus empty vector or STK31-expressing vector using Lipofectamine 2000. After 48 and $72 \mathrm{~h}$ incubation at $37^{\circ} \mathrm{C}, 5 \% \mathrm{CO}_{2}$, supernatant containing the lentivirus was collected from 293T cells and centrifuged at $250 \mathrm{x}$ g for $2 \mathrm{~min}$ at room temperature, then filtered and applied to target cells. For lentiviral infection, cells $\left(3 \times 10^{5}\right)$ for KD experiments or ectopic expression were seeded onto 6-well culture plates before addition of viral supernatant. After $72 \mathrm{~h}$, the medium was changed to RPMI-1640 medium containing $1 \mu \mathrm{g} / \mathrm{ml}$ puromycin (Sigma-Aldrich; Merck KGaA). After 2 weeks of puromycin selection, the change in expression levels was confirmed by RT-qPCR (as aforementioned) and western blotting.

Western blotting. For protein extraction from GC cell lines, RIPA lysis buffer (Invitrogen; Thermo Fisher Scientific, Inc.) with protease inhibitor cocktail (Sigma-Aldrich; Merck KGaA) was used and then concentration was quantified via Bradford Protein Assay (Bio-Rad Laboratories, Inc.) Protein samples (50 $\mu \mathrm{g} /$ lane) were loaded onto $10 \%$ acrylamide gel. Electrophoresis was performed using a Bio-Rad Western Blotting system. (Bio-Rad Laboratories, Inc.) Proteins were transferred to a polyvinylidene fluoride membrane (Sigma-Aldrich; Merck KGaA) and blocked in 5\% skimmed milk in Tris-buffered saline $(0.1 \%$ Tween-20) for $30 \mathrm{~min}$ at room temperature. The membranes were incubated with primary antibodies $(1: 1,000)$ at $4^{\circ} \mathrm{C}$ overnight. The antibodies were as follows: Anti-STK31 (cat. no. ab155172; Abcam), anti-Caspase 3 (cat. no. 9662; Cell Signaling Technology, Inc.), anti-PARP (cat. no. 9542; Cell Signaling Technology, Inc.) and anti-Tubulin (cat. no. T5168; Sigma-Aldrich; Merck KGaA), then Probed with mouse anti-rabbit IgG conjugated with horseradish peroxidase (1:5,000; cat. no. sc-2357; Santa Cruz Biotechnology, Inc.). Immunopositive bands were visualized using an enhanced luminescence image analyzer LAS-4000 (FUJIFILM Wako Pure Chemical Corporation) and the intensity for each band was estimated by Image J version 1 software (National Institutes of Health).
Cell proliferation assay. For cell proliferation assays, $1 \times 10^{3}$ cells were plated in a 96-well plate and proliferation was measured with the EZ-Cytox Cell Viability Assay kit (Itsbio) using a microplate reader (Molecular Devices, LLC) at $450 \mathrm{~nm}$. For colony-forming assays, $1 \times 10^{3}$ cells were plated in a 6-well plate. RPMI-1640 Media (Welgene Inc.) were replaced every 3 days then cells were incubated at $37^{\circ} \mathrm{C}$. After 2 weeks, colonies were stained with crystal violet solution ( 0.5 crystal violet, 3.7 formaldehyde, $30.0 \%$ ethanol) for $2 \mathrm{~h}$ at room temperature, and the number of viable cells was manually counted in each well. All assays were performed in triplicate.

Cell migration assay. Transwell migration assays were performed in a 24-well Transwell chamber (Corning, Inc.) fitted with a polycarbonate membrane (pore size, $8 \mathrm{~mm}$ ). Cells were suspended in $100 \mu \mathrm{l}$ serum-free RPMI-1640 medium (Welgene, Inc.), and $2 \times 10^{4}$ cells were seeded in the upper chamber. The lower chamber was filled with RPMI-1640 medium containing $10 \%$ fetal bovine serum. After 16-24 h incubated at $37^{\circ} \mathrm{C}$, migrated cells were stained for $2 \mathrm{~h}$ at room temperature with $0.5 \%$ crystal violet solution. A total of three independent fields of view were observed using a fluorescence microscope (magnification, x10) for each membrane, and migrated cells were manually counted in each field.

Cell cycle analysis. MKN1 cells were treated $0.05 \%$ trypsin for $3 \mathrm{~min}$ at $37^{\circ} \mathrm{C}$ and then harvested at a density of $2 \times 10^{6}$ per ml. Cells were washed with ice-cold PBS and fixed with $70 \%$ ethanol for $24 \mathrm{~h}$ at $4{ }^{\circ} \mathrm{C}$. Prior to analysis, cells were stained with $50 \mu \mathrm{g} / \mathrm{ml}$ propidium iodide solution for $2 \mathrm{~min}$ at room temperature. Cell cycle analysis was performed using a flow cytometer (FacsCalibur; BD Biosciences) with blue laser $488 \mathrm{~nm}$ and FlowJo 10.7.1 and Cell Quest Software (BD BioSciences).

Gap closure assay. Mobility of STK31-expressing MKN74 cells was measured using gap closure assay (Ibidi Gmbh). Cell suspension at a density of $1 \times 10^{6}$ per $\mathrm{ml}$ in $70 \mu \mathrm{l}$ volume were seeded in Cultured-Inserts 2-well (diameter, $35 \mathrm{~mm}$ ). Following incubation at $37^{\circ} \mathrm{C}$ for $24 \mathrm{~h}$, the Culture-Insert was detached from the well using forceps and filled with serum-free RPMI-1640 medium. Cell images were captured after 24, 48, 72 and $96 \mathrm{~h}$ under a fluorescence microscope (magnification, $\mathrm{x} 10)$. The area of gap closure in three fields of view was calculated with ImageJ software (v.1.8.0; National Institutes of Health).

Xenograft assay. A total of 10 female BALB/C nude mice

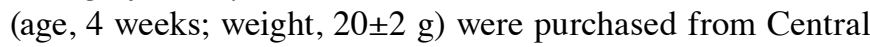
Animal Laboratory (Shizuoka, Japan) and maintained with regular mouse chow and water at a constant temperature of $22 \pm 1^{\circ} \mathrm{C}$ and $50-60 \%$ humidity under a 12-h light/dark cycle and specific pathogen-free conditions for the xenograft assay. Mice were anesthetized before injection using isoflurane (3-4\% oxygen) to minimize pain. After 1 week, parental or STK31-expressing MKN74 cells $\left(5 \times 10^{6}\right.$ cells per mouse) were subcutaneously injected into nude mice. Mice were monitored daily to check sickness and feed daily and to determine weight loss $>20 \%$ twice a week. Once palpable tumors devloped, tumor size was measured using Vernier calipers and tumor volume 
was calculated according to the following formula: Volume $\left(\mathrm{mm}^{3}\right)=$ width $^{2} \times$ length $/ 2$. All mice were euthanized using $30-70 \%$ volume/min $\mathrm{CO}_{2}$ in chamber at day 53 . The humane endpoints were when the largest tumor size exceeded $20 \mathrm{~mm}$ or feed intake or drinking water were affected due to necrosis, infection or ulcer. None of the mice died before endpoints of the study. Experiments using mouse were conducted under the Institutional Animal Care and Use Committee-approved protocols at KRIBB in accordance with institutional guidelines (approval no. KRIBB-AEC-15102).

Public data. The 450K HumanMethylation BeadChip data (accession number GSE103186) for 39 gastric mucosae and 76 IM tissue samples from GC-free patients (18) were downloaded to compare methylation status in the STK31 promoter. Gene expression and 450K HumanMethylation BeadChip data for 230 primary GCs and 450K HumanMethylation BeadChip data for two normal tissue samples (19) were downloaded from The Cancer Genome Atlas (TCGA) portal (portal.gdc.cancer.gov/) and the National Center for Biotechnology Information Gene Expression Omnibus (ncbi. nlm.nih.gov/geo/). Because methylation data for normal tissue are limited in the TCGA database, additional data for 10 gastric mucosa samples were obtained public data [accession nos. GSE50192 $(n=4)$ and GSE31848 $(n=6)](20,21)$ produced by the same platform. In order to assess the activation status of the STK31 promoter in primary GCs, public data (accession no. GSE51776) for histone modifications produced by nano-scale chromatin immunoprecipitation-sequencing (Nano-ChIP-seq) of paired GC and non-tumor tissue was downloaded (22). The downloaded sequence reads were quality controlled using cut-adapt (v1.1) on a public site (github.com/marcelm/cutadapt/). Quality-control parameters were read for quality $>30$ (Phred score), read length $>20 \mathrm{bp}$ and replicated level $<40 \%$. The sequence reads were mapped with bowtie2 (v2.2.2) using default parameters $(\mathrm{m}=1)$. Duplicate reads were removed using the picard (v.2.18.14) markduplicates function. Unique reads were identified by peak calling using macs2 (v.2.0.5) with default parameters. For predicting patient outcomes, gene expression data (accession no. GSE26253) for GC ( $\mathrm{n}=432)$ in the Samsung Medical Center cohort (SMC) (23) was downloaded from the Gene Expression Omnibus database. The samples included 68 stage I, 167 stage II, 130 stage III and 66 stage IV tumors and one non-information tumor, taken from 152 females and 280 males (average age, 52 years). All samples were obtained with written informed consent, and their use was approved by the Internal Review Board of the SMC, Seoul, South Korea (approval no. SMC 2010-10-025).

Statistical analysis. A paired t-test was used to examine differences in mRNA levels and methylation between paired gastric tumor and adjacent non-tumor tissues. Values are expressed as the mean \pm SD of $\geq 3$ independent repeats.. Correlations between SKT31 expression levels and CpG methylation was determined using Pearson's correlation coefficient. For the comparison of multiple groups of GC type from TCGA data, pairwise P-values were calculated using the Wilcoxon rank-sum the Student's t-test, respectively, and were corrected for multiple comparison by Bonferroni method $(n=3)$. For comparison of of STK31 mRNA levels in GC cells following drug treatment, pairwise P-values were calculated using paired Student's t-test and was corrected for multiple comparison by Bonferroni method $(\mathrm{n}=3)$. For in vitro assays of STK31 expression levels in STK31-KD cells, pairwise P-values were calculated using paired Student's t-test and were corrected for multiple comparison by Bonferroni method $(n=2)$. Kaplan-Meier survival analysis with log-rank test was used to estimate the difference in overall survival between STK31 high and low expression groups in combined cohorts of CNUH and SMC. All statistical analysis was performed using the $\mathrm{R}$ statistical programming language (Version 4.0.2; r-project.org/).

\section{Results}

STK31 is an early-onset target for hypomethylation in $G C$ development. Our previous study (8) identified 174 hypomethylated promoters in GC cells (91 GC-specific and 83 early-onset) via methylome analysis with laser-capture microdissected cells of a single patient with intestinal-type GC (IGC). Early-onset hypomethylation was defined as a methylation difference $>2$-fold in IM and GC compared with gastric mucosa cells. STK31 was an early-onset hypomethylated targets (Fig. 1B). RRBS data from the UCSC Genome Browser (hg19) revealed that $\mathrm{CpG}$ methylation signatures (purple vertical lines) at the STK31 promoter were predominant in GM cells but mostly absent in IM and completely absent in GC (Fig. 1B).

CpG hypomethylation of the STK31 promoter is associated with STK31 upregulation in primary GC. RT-qPCR analysis was performed with four paired gastric tumor and adjacent non-tumor tissues, revealing that STK31 was silenced in non-tumors but expressed in all tumor samples tested (Fig. 2A). Bisulfite sequencing analysis with three of four paired clinical tissues revealed 51.4-69.4\% methylation in Region 1 of non-tumors but $16.7-30.3 \%$ in paired tumors (Fig. 2B). For Region 2, the difference was also significant (non-tumor, 92.7-96.4\%; tumor, 64.8-86.1\%; Fig. 2B), although not as large as for Region 1. These data suggested that STK3I expression levels in tumors may be a consequence of $\mathrm{CpG}$ hypomethylation at the promoter comprising Regions 1 and 2 .

STK31 expression levels and promoter methylation were analyzed using 145 paired clinical tissue samples of the CNUH cohort. RT-qPCR analysis of STK31 revealed significant upregulation in tumor $(16.70 \pm 3.60 \%)$ compared with non-tumor samples $(13.29 \pm 5.49 \%$; Fig. $2 \mathrm{C} ; \mathrm{P}=0.02)$. A a significant increase in expression was defined as $>2$-fold with respect to the values for tumors compared with paired non-tumors; increased STK31 expression was apparent in approximately half (72/145) of tumors. Methylation at two CpG sites, namely CpG\#23 and \#24, within Region 2 was quantified by pyrosequencing (Fig. 1C) of 120 paired clinical tissues and compared with the corresponding RT-qPCR data. Pyrosequencing of these two sites revealed $92.1 \pm 6.4 \%$ methylation in non-tumors and $79.7 \pm 13.0 \%$ in tumors, the difference for which was significant (Fig. 2D; $\mathrm{P}<2.2 \times 10^{-16}$ ). Finally, there was a negative correlation between methylation at the two CpG sites and STK31 expression in 120 matched tumors (Fig. 2E; $\mathrm{r}=-0.34 ; \mathrm{P}=1.1 \times 10^{-4}$ ). 


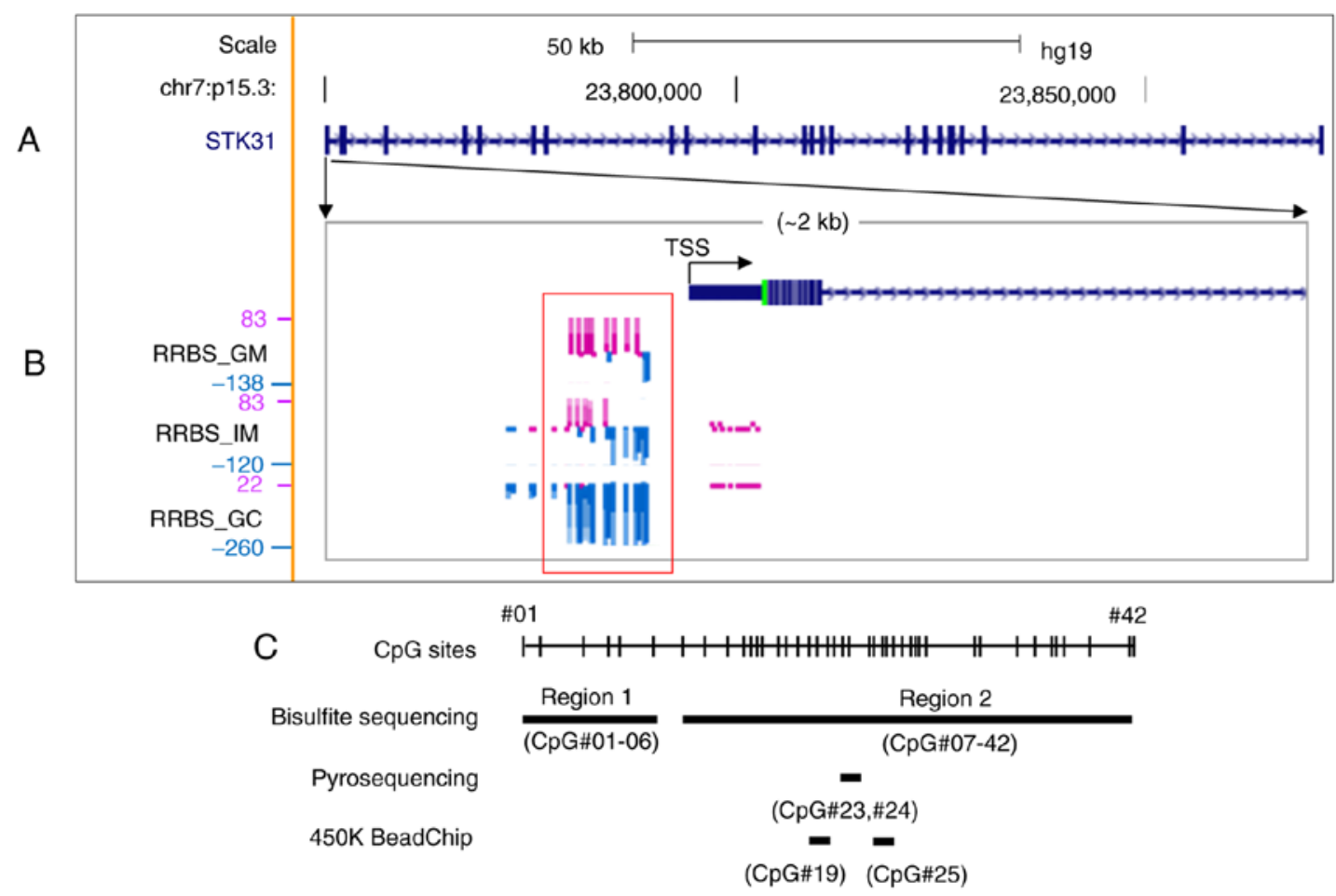

Figure 1. Methylation profile of the STK31 promoter in cells of a clinical tissue isolated by laser capture microdissection. (A) Gene structure of STK31 on human chromosome 7p15.3. The map was modified from the UCSC Genome Browser (hg19, genome.ucsc.edu). The distance from TSS to transcription end site is $\sim 122.4 \mathrm{~kb}$. Thick black bars denote exons. (B) RRBS methylome profiles in an enlargement of the $S T K 31$ promoter region ( $\sim 2 \mathrm{~kb})$ in paired GM, IM and GC cells by mirroring the UCSC Genome Browser. The height of each vertical line indicates methylation score for individual CpGs. Methylation and non-methylation scores are displayed as purple and blue bars, respectively. The red rectangle highlights differentially methylated region in GM compared with IM or GC. (C) Strategy for analysis. Bisulfite sequencing was performed for Regions 1 (6 CpGs, -386 to -198 nucleotides from TSS) and 2 (39 CpGs, - 171 to 249 nucleotides). Pyrosequencing was performed for CpG\#23 (+54 nucleotidea from TSS) and \#24 (+58 from TSS). Positions of CpG probes CpG\#19 (cg05000488, -46 from TSS) and CpG\#25 (cg11755819, +67 from TSS) are shown, proximal to the STK31 TSS from 450K HumanMethylation BeadChip. STK, serine/threonine kinase; TSS, transcription start site; RRBS, reduced representation bisulfite sequencing; GM, gastric mucosa; IM, intestinal metaplasia; GC, gastric cancer.

From the public data for $450 \mathrm{~K}$ HumanMethylation BeadChip of the TCGA, methylation status between GC and non-tumors at $\mathrm{CpG}$ sites proximal to the STK31 promoter was compared; the promoter and upstream region were hypomethylated in primary GC, whereas there was no difference in $\mathrm{CpG}$ methylation on the gene body (Fig. 3A). For example, methylation at $\operatorname{cg} 05000488$ within the $S T K 31$ promoter was significantly decreased in GC, especially in IGC (Fig. 3B), whereas $S T K 31 \mathrm{mRNA}$ expression levels were significantly increased in GC (Fig. 3C), revealing a negative correlation between CpG methylation and STK31 mRNA expression levels (Fig. 3D). In addition, methylation status was compared at the same $\mathrm{CpG}$ sites proximal to the STK31 promoter from the public data (GSE103186) for 450K BeadChip of gastric mucosae and IM tissue from GC-free patients (17). Methylation at $\operatorname{cg} 05000488$ within the STK31 promoter was significantly decreased in IM compared with that in gastric mucosae (Fig. 3E).

Change in promoter methylation in GC cell lines alters STK31 expression. In order to investigate the association between STK31 expression and methylation of its promoter in GC cell lines, RT-qPCR and bisulfite sequencing analysis of GC cell lines were performed. Based on the RT-qPCR results, cell lines were divided into two groups according to the median relative STK31 expression level. Fig. 4A and B); the STK31-expressing group (+; >median value) included SNU484, SNU520,
KATOIII and MKN1 and the weakly/non-expressing group (-; <median value) included SNU001, SNU638, SNU719 and MKN74. For Region 1, bisulfite sequencing revealed that members of the STK31 (+) group, except for SNU484, exhibited low mean methylation (12.1-69.1\%), whereas the STK31 (-) group had relatively high methylation (81.2-92.6\%; Fig. 4B). In Region 2, however, members of both groups were highly methylated (86.8-99.1\%; Fig. 4B). The association between methylation status at two $\mathrm{CpG}$ sites (CpG\#23 and \#24) and STK31 expression was examined in both groups; methylation status tended to be decreased in STK31 (+) group compared with the STK31 (-) group and STK31 expression levels tended to be increased in STK31 (+) group compared with the STK31 (-) group (Fig. 4C) in both bisulfite sequencing and pyrosequencing analyses. In order to determine whether STK31 expression is controlled epigenetically, cells were treated with 5-aza-dC and/or TSA. Treatment with 5-aza-dC significantly restored STK31 expression levels in SNU-638, SNU-719, and MKN74 cells (Fig. 4D). Treatment of GC cells with both 5-aza-dC and TSA also restored STK31 expression levels (Fig. 4D), suggesting that its expression in GC cells may be regulated epigenetically.

Genome-wide histone modification data reveal upregulation of the STK31 promoter in primary GC. From the public data (GSE51776), unique reads were identified by peak calling using macs2 with default parameters. This yielded peak regions for 
A
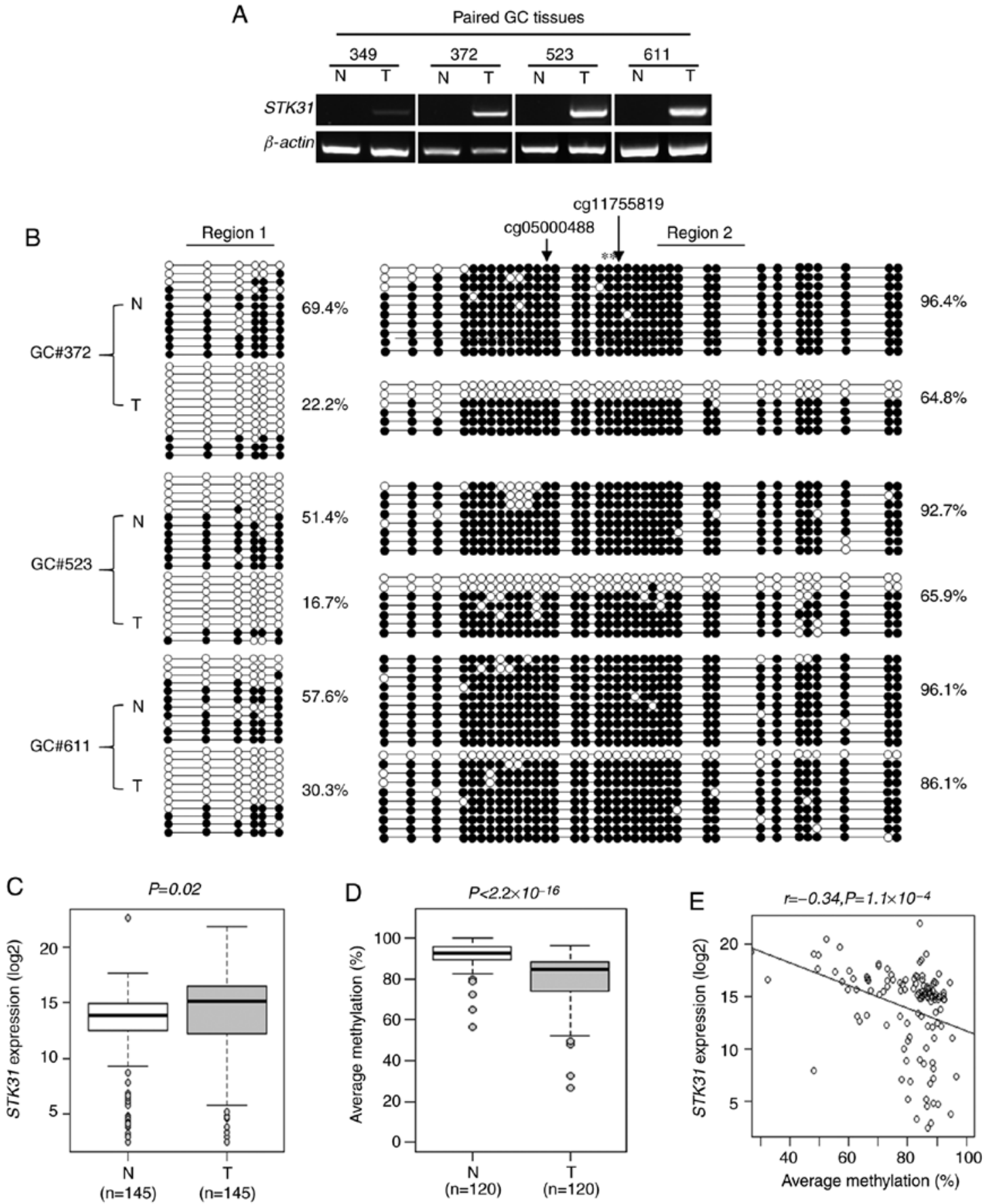

Figure 2. STK31 expression and bisulfite sequencing analysis of clinical tissue samples. (A) RT-qPCR analysis of STK31 in four paired gastric T and N tissue samples. $\beta$-actin was used as an internal control. (B) Bisulfite sequencing analysis was performed with three paired GC and $\mathrm{N}$ tissue samples. Black and white circles indicate methylated and non-methylated $\mathrm{CpG}$ sites, respectively. Each row represents a single clone. Mean percentages of CpG sites methylated in each sample are shown. Asterisks indicate $\mathrm{CpG}$ sites (CpG\#23 and \#24) used for pyrosequencing. Arrows indicate CpG probes from 450K BeadChip. (C) STK31 expression in 145 paired $\mathrm{N}$ and GC samples from the CNUH cohort. RT-qPCR was performed and expression levels were normalized to $\beta$-actin. (D) STK31 methylation in paired samples from the CNUH cohort. Pyrosequencing was performed at two CpG sites. The average value for methylation was calculated for each sample. (E) Pearson's correlation analysis between STK31 methylation and expression levels in the CNUH cohort. STK, serine/threonine kinase; RT-q, reverse transcription-quantitative; T, tumor; N, non-tumor; CNUH, Chungnam National University Hospital.

H3K4me3 (chromatin mark for active promoters), H3K4me1 (chromatin mark for active enhancers and promoters) and H3K27ac (chromatin mark for active regulatory elements), for which five paired normal and gastric tumor tissue samples were merged. Then, signatures for promoter activity were examined near the STK31 promoter. Gain of STK31 promoter activity (increased $\mathrm{H} 3 \mathrm{~K} 4 \mathrm{me} 3$ and $\mathrm{H} 3 \mathrm{~K} 27 \mathrm{ac}$ ) was evident in primary GC but not in normal mucosae (Fig. 5).
STK31 KD inhibits cell proliferation and migration and induces $G_{l}$ arrest in vitro. It was next investigated whether STK31 expression in SNU484 or MKN1 cells, in which STK31 was highly expressed (Fig. 6A), could be knocked down by two shRNAs. RT-qPCR and western blotting analysis confirmed that STK31 expression levels significantly decreased in STK31-KD SNU484 and MKN01 cells (Fig. 6A). Each shRNA significantly decreased colony formation (Fig. 6B), 

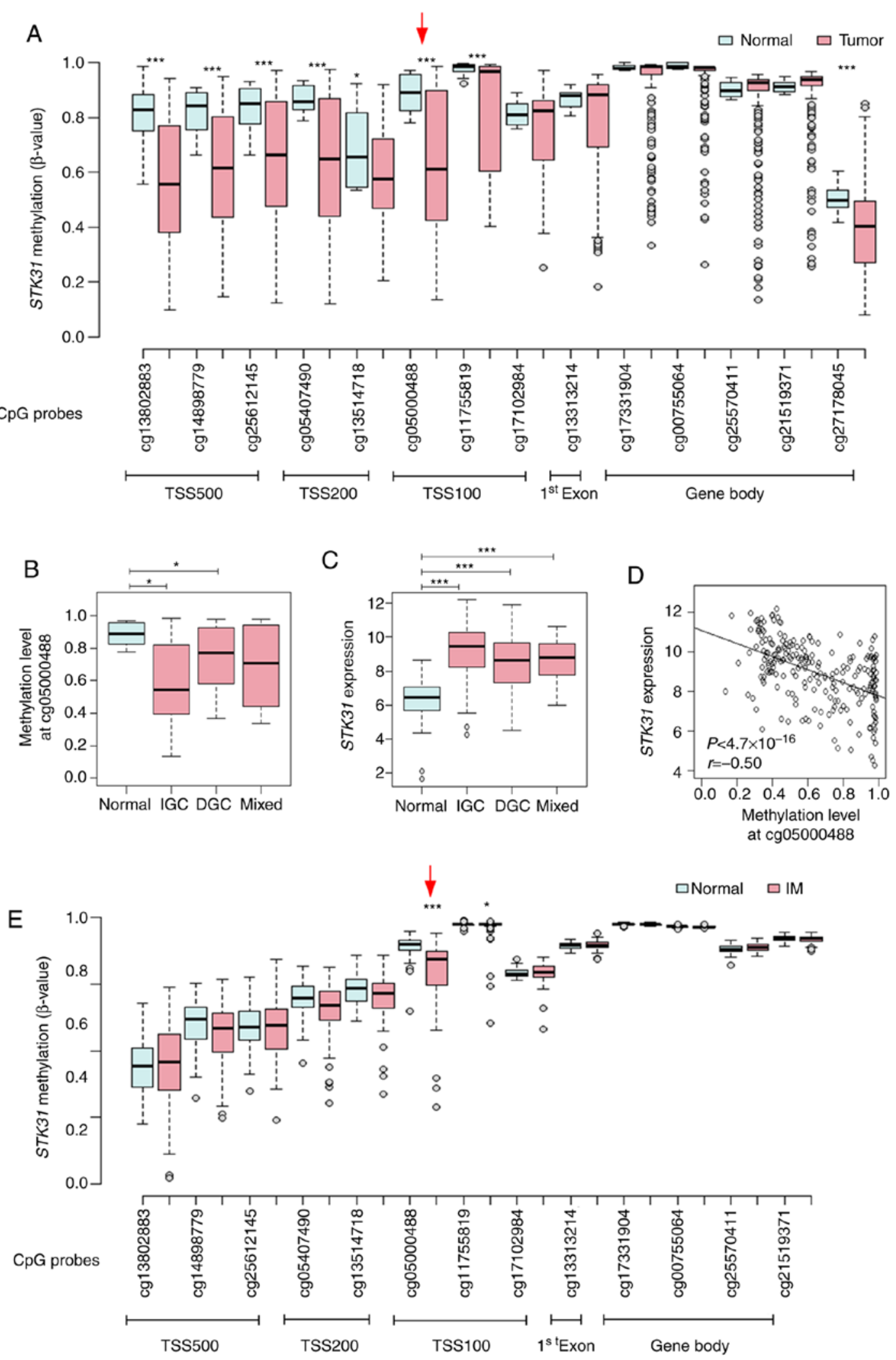

Figure 3. Correlation between STK31 promoter methylation and expression levels in primary gastric tumors or STK31 promoter methylation in IM from the public database. (A) Methylation status at CpG sites proximal to the STK31 promoter. $\beta$-values at $14 \mathrm{CpG}$ sites from TSS500, TSS200, TSS100, exon 1 and the gene body were retrieved from 450K HumanMethylation BeadChip data for 29 gastric mucosa samples (normal) and 214 gastric tumors including IGC $(\mathrm{n}=140)$, DGC $(\mathrm{n}=57)$ and mixed-type GC $(\mathrm{n}=17)$ from the TCGA database. Red arrow indicates cg05000488, the CpG site in TSS100 at which correlation with STK31 expression levels was examined. (B) STK31 methylation status at cg05000488 was examined in normal tissue, IGC, DGC and mixed-type GC. P-values were determined using the Wilcoxon rank-sum test and corrected for multiple comparisons by Bonferroni method (n=3). (C) STK31 expression was examined. Pairwise P-values were calculated using Student's t-test and corrected for multiple comparisons by Bonferroni method (n=3). (D) Pearson's correlation analysis between methylation at cg05000488 and STK31 expression levels in the TCGA cohort. (E) Methylation status at CpG sites proximal to the STK31 promoter in IM. $\beta$-values at $13 \mathrm{CpG}$ sites were retrieved from 450K BeadChip data for 39 normal and 76 IM samples from public data GSE103186 (17). P-values were determined using Student's t-test. ${ }^{*} \mathrm{P}<0.05,{ }^{* * *} \mathrm{P}<0.001$. STK, serine/threonine kinase; IM, intestinal metaplasia; TSS, transcription start site; IGC, intestinal-type gastric cancer; DGC, diffuse-type gastric cancer; TCGA, The Cancer Genome Atlas. 
A

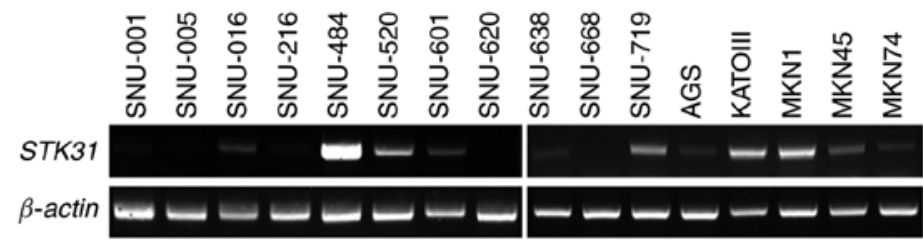

B Region 1

Region 2

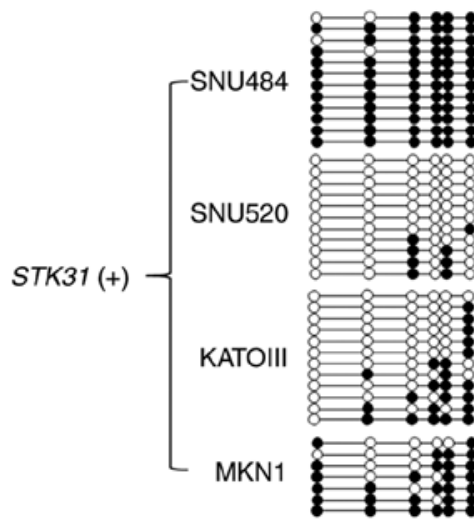
$94.4 \%$

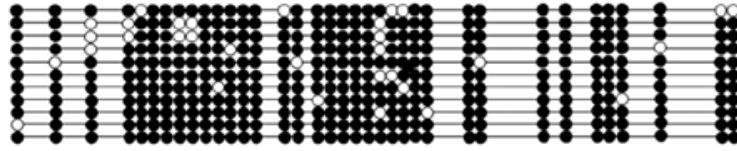

$91.9 \%$

$12.1 \%$

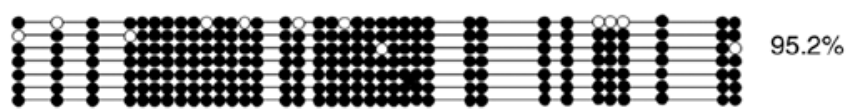

$31.2 \%$
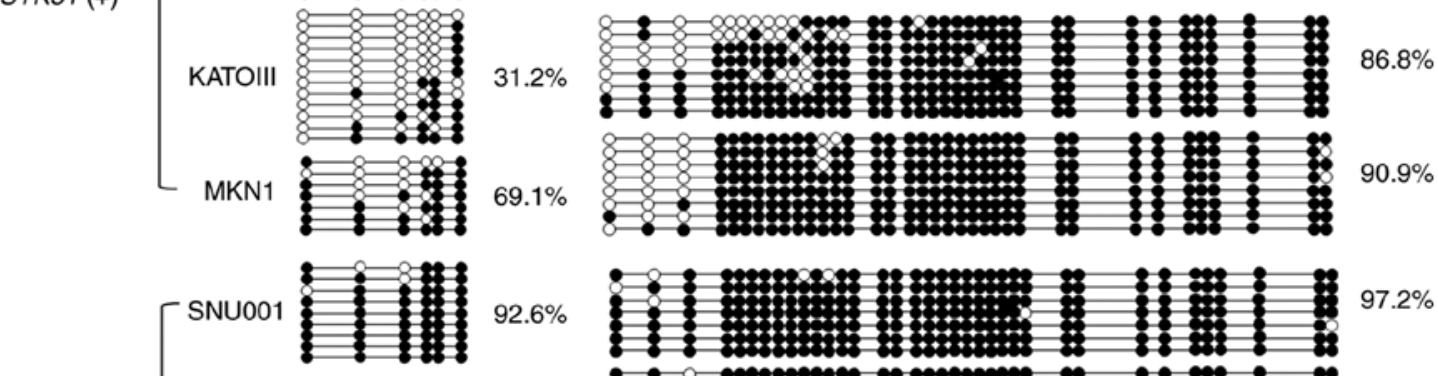

$92.6 \%$
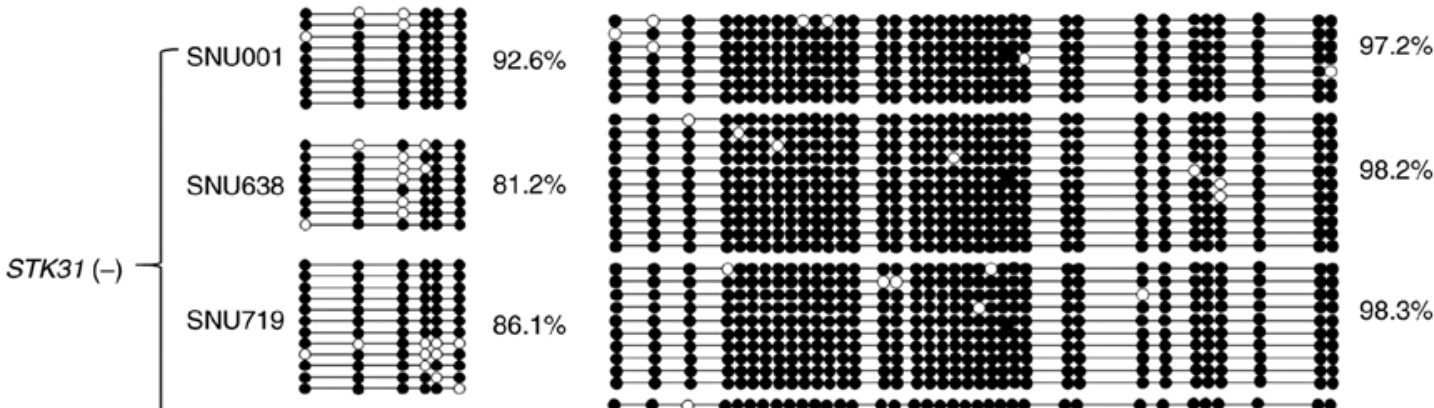

$81.2 \%$

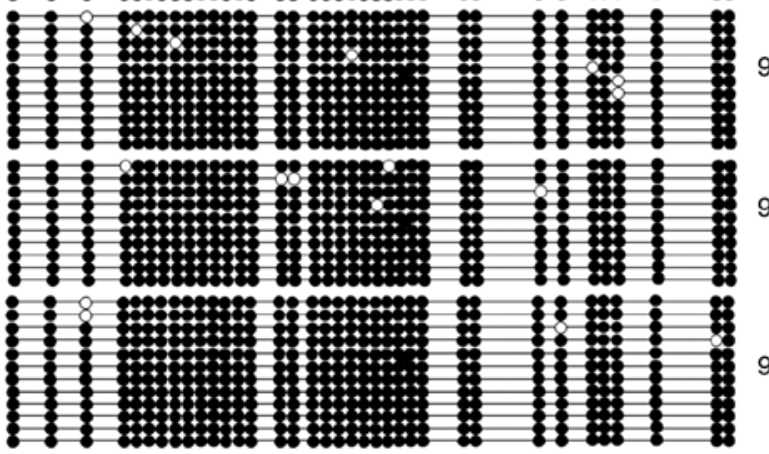

MKN74

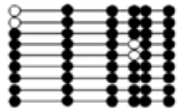

$92.6 \%$

\section{$86.1 \%$}

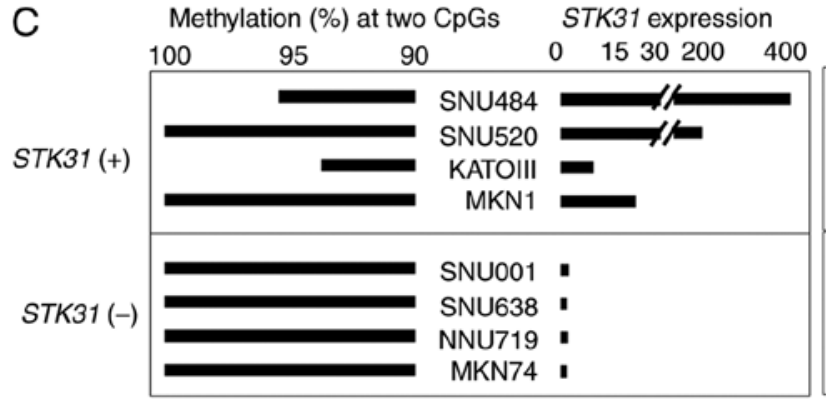

Methylation (\%) at two CpGs

STK31 expression
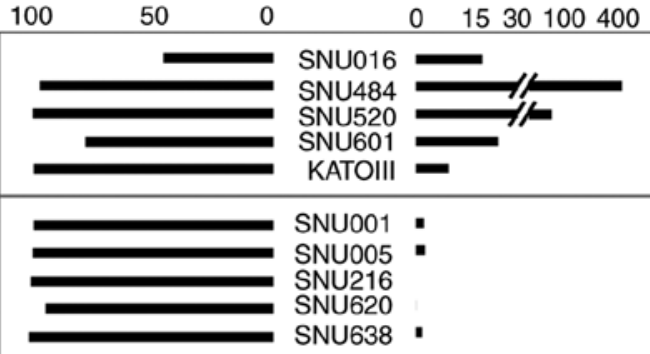

D
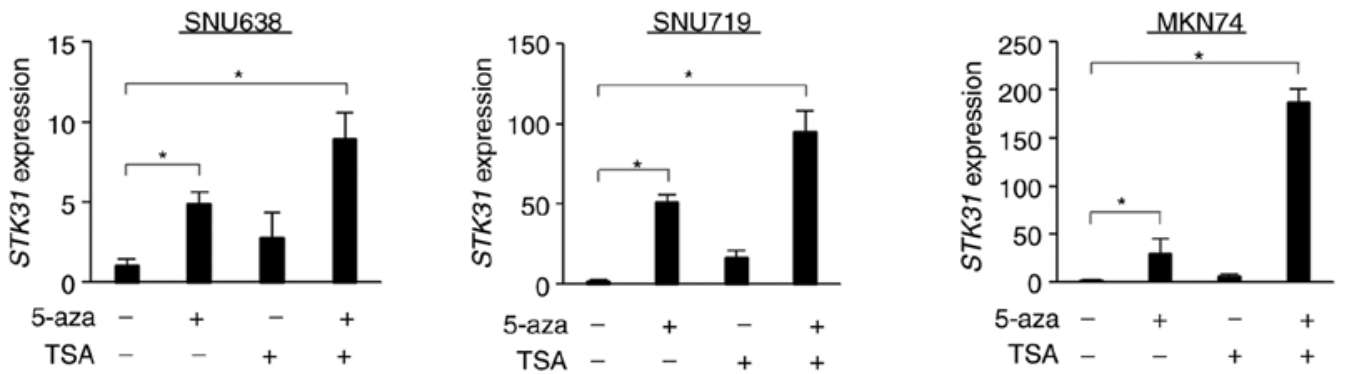

Figure 4. STK31 expression and bisulfite sequencing analysis of GC cell lines. (A) RT-qPCR analysis of 16 GC cell lines. (B) Analysis of bisulfite sequencing A total of eight GC cell lines were categorized based on relative STK31 expression (determined by RT-qPCR) as strong (+) or weak/silenced (-). (C) Association between STK31 expression and mean methylation at CpG\#23 and \#24. Methylation status was based on bisulfite sequencing and pyrosequencing analysis. (D) Restoration of STK31 mRNA levels following treatment with 5-aza and/or TSA. STK31 expression levels were assessed by RT-qPCR and normalized to $\beta$-actin. Data are presented as the mean $\pm \mathrm{SD}$ of three independent experiments. Pairwise P-values were calculated using Student's t-test and corrected for multiple comparison by Bonferroni method. *P<0.05. STK, serine/threonine kinase; GC, gastric cancer; RT-q, reverse transcription-quantitative; 5-aza, 5-aza-2-deoxycytidine; TSA, trichostatin A. 


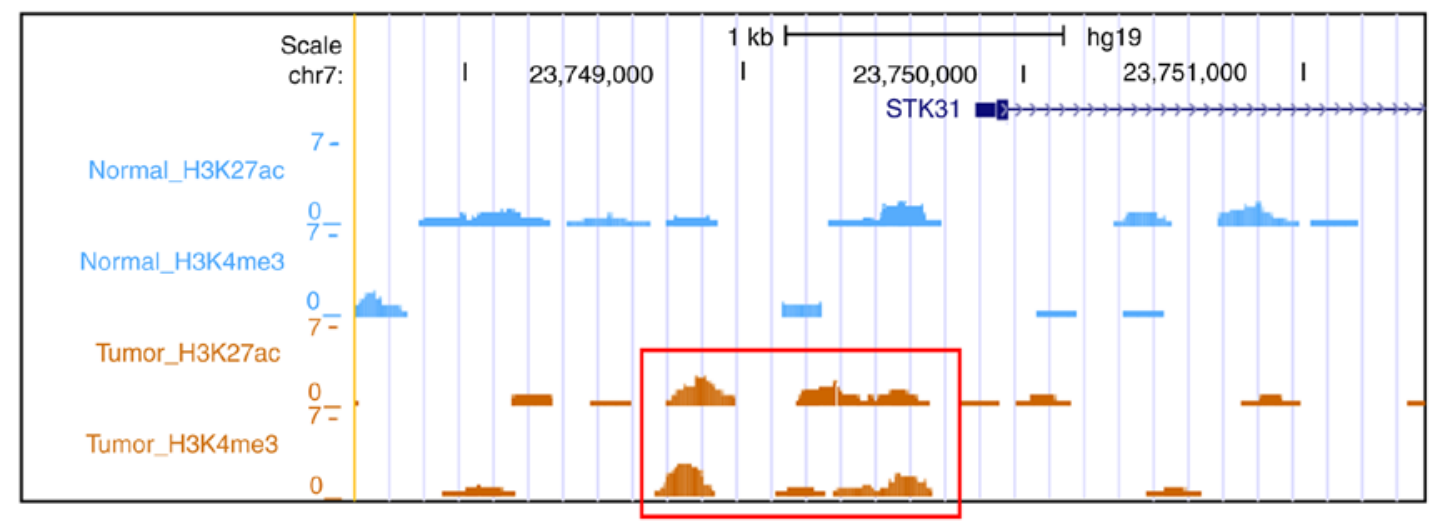

Figure 5. Histone modifications at STK31 promoters in paired normal and GC tissue. Public data for histone modifications in paired tissue, as determined by nano-scale chromatin immunoprecipitation-sequencing (22), were downloaded and processed. H3K4me3 and H3K27ac peak regions from five paired normal and gastric tumor tissue samples were merged and visualized with the UCSC Genome Browser (hg19). Red rectangle highlights gain of promoter activity with increased $\mathrm{H} 3 \mathrm{~K} 27 \mathrm{ac}$ and $\mathrm{H} 3 \mathrm{~K} 4 \mathrm{me} 3$ at each promoter in primary GC. STK, serine/threonine kinase; GC, gastric cancer.

A
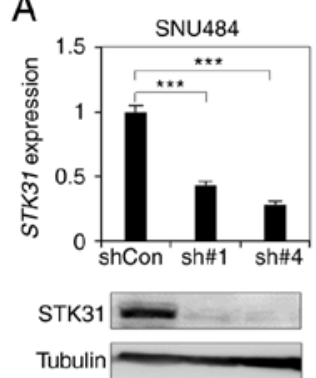

C
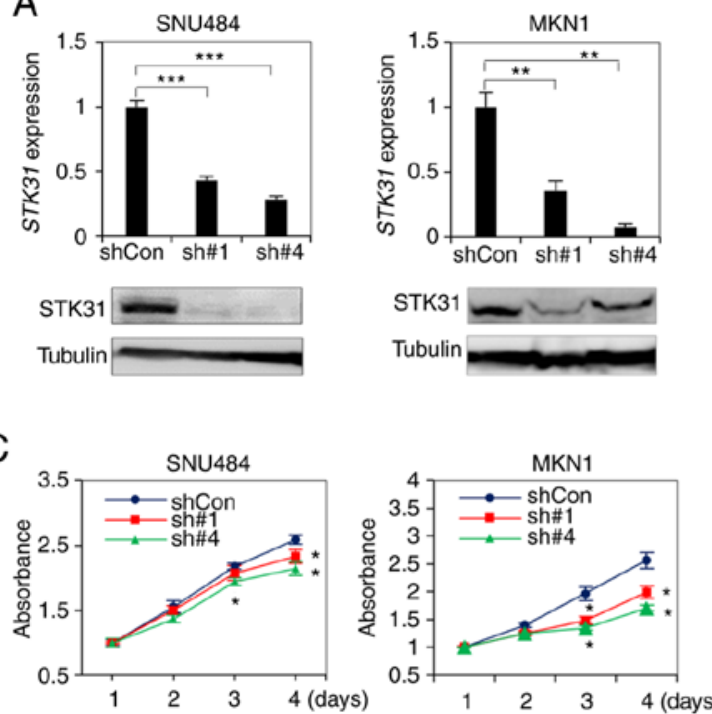

B
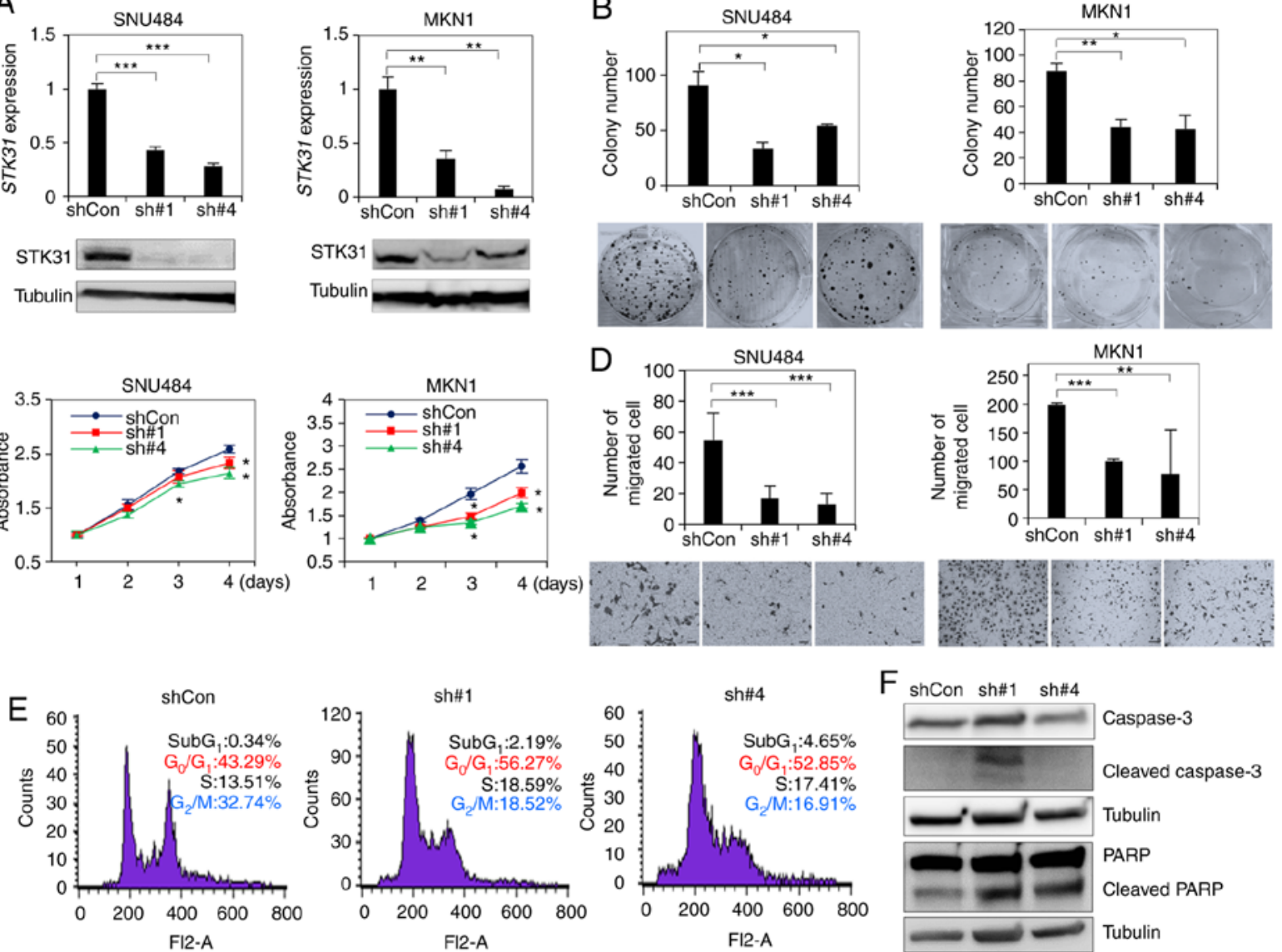

$F_{\text {shCon }}$ sh\#1 sh\#4

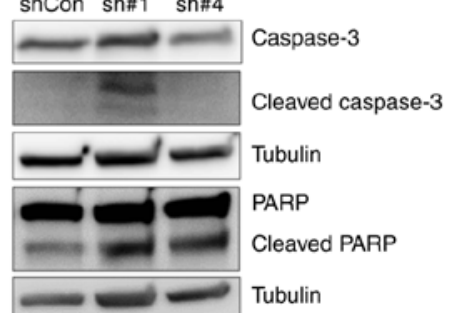

Figure 6. In vitro assay of STK31 expression levels in STK31-KD cells. (A) Establishment of STK31-KD cells by expression of shRNA. SNU484 and MKN01 cells were transfected with either of two lentiviral STK31 shRNAs (sh\#1, sh\#4) or scrambled-sequence shCon and cultured for 2 weeks. KD and control cells were compared by reverse transcription-quantitative PCR and western blotting. Tubulin was used as an internal control. (B) Colony formation assay. Transfected cells were plated on 6-well plates at $1 \times 10^{3}$ cells per well. After 2 weeks, colonies were stained with crystal violet and counted. (C) Relative viability of STK31-KD cells over 4 days was measured using EZ-Cytox Cell Viability Assay kit and compared with empty vector control (PLKO). (D) Migration assay. Transfected cells were plated on Transwell chambers at 2x104 cells per well. After 18-22 h, Transwell membranes were stained with crystal violet and cells were counted. (E) Cell cycle analysis of STK31-KD MKN1 cells. Following PI staining, cells were assessed by flow cytometry. (F) Western blot analysis of caspase-3 and PARP cleavage in MKN1 cells. Two membranes were used for PARP and Caspase-3. Pairwise P-values were calculated using Student's t-test and corrected for multiple comparison by Bonferroni method $(n=2) .{ }^{*} \mathrm{P}<0.05,{ }^{* *} \mathrm{P}<0.01,{ }^{* * * *} \mathrm{P}<0.001$. STK, serine/threonine kinase; KD, knockdown; sh, short hairpin; Con, control.

proliferation (Fig. 6C) and migration (Fig. 6D) of both STK31-KD cell lines compared with cells treated with control shRNA. In order to investigate the underlying mechanisms for the cell proliferation of STK31 suppression, the effect of STK31 silencing on cell cycle progression in MKN1 cells was assessed by flow cytometry. STK31-KD MKN1 cells was increased in 
A

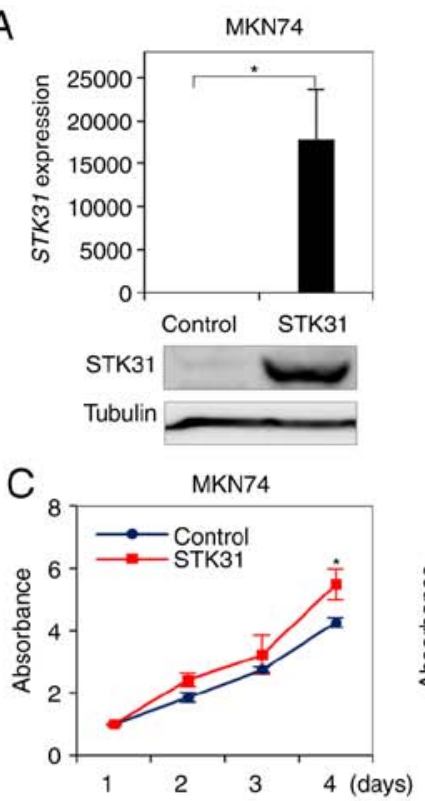

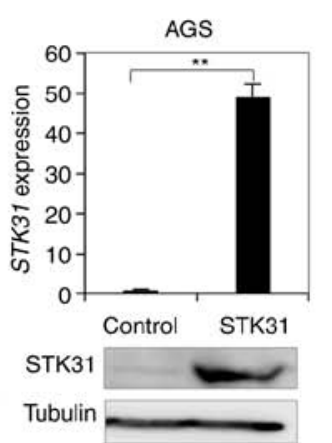

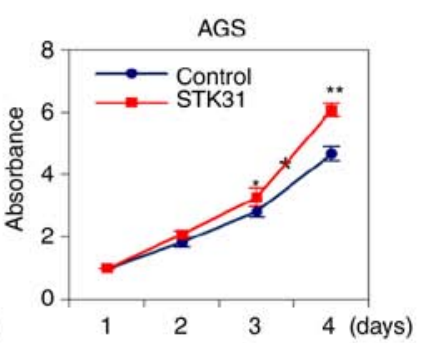

B
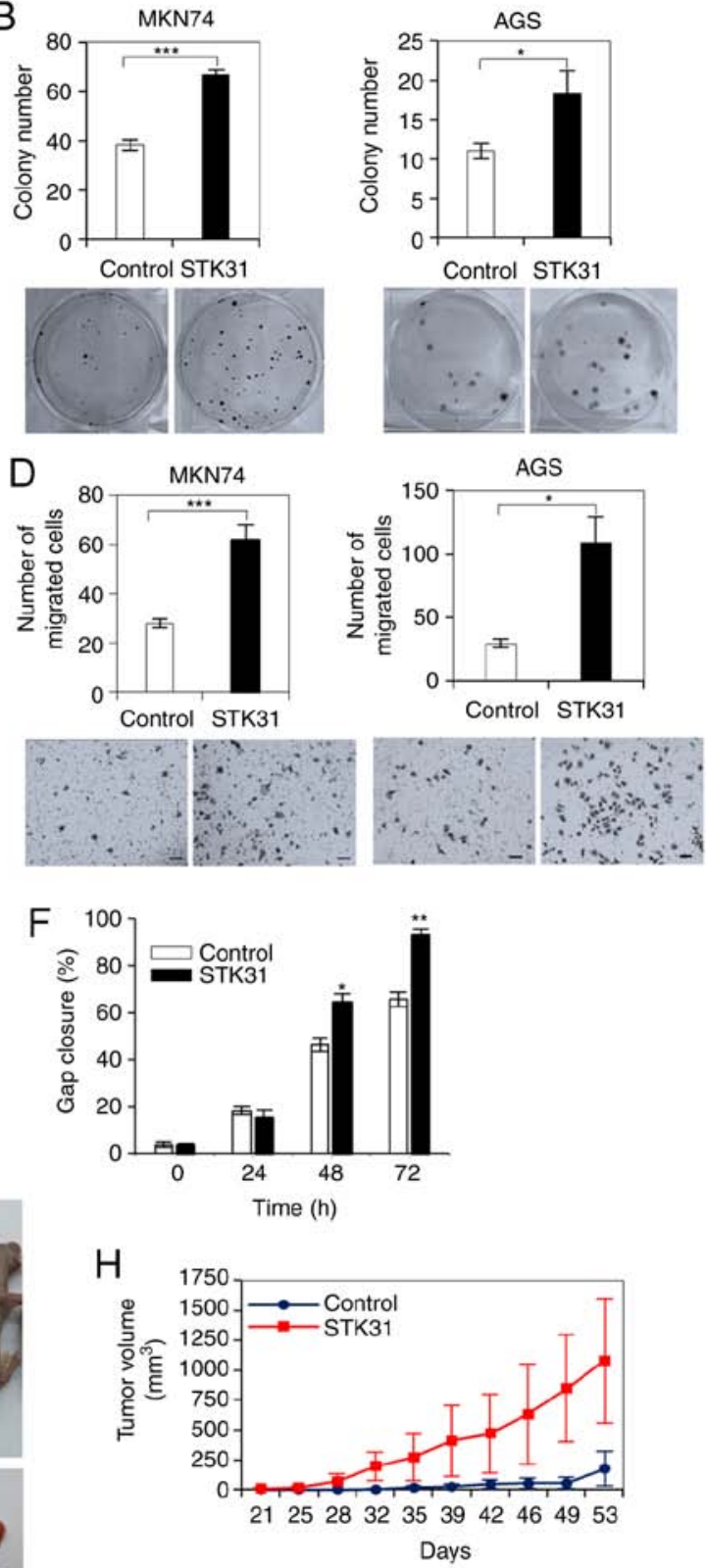

Figure 7. In vitro and in vivo assay of STK31 expression in cells ectopically expressing STK31. (A) Establishment of STK31-expressing cells. Gastric cancer cell lines MKN74 and AGS were transfected with lentiviral STK31-expression vector. STK31 mRNA and protein expression levels was compared with cells transfected with an empty vector control (pCDH). Tubulin was used as an internal control. (B) Colony formation, (C) proliferation and (D) migration assays were performed. (E and F) Gap closure assay following STK31 overexpression in MKN74 cells at a density of 1x10 cells. Scale bar, $100 \mu \mathrm{m}$. (G) Xenograft assay with transfected MKN74 cells. Mice were sacrificed at day 53 and tumor volumes were measured. Scale bar, $1 \mathrm{~cm}$. (H) Tumor growth curves for STK31-expressing and control MKN74 cells in nude mice. P-values were determined using Student's t-test. ${ }^{*} \mathrm{P}<0.05,{ }^{* *} \mathrm{P}<0.01,{ }^{* * * *} \mathrm{P}<0.001$. STK, serine/threonine kinase.

the $\mathrm{G}_{0} / \mathrm{G}_{1}$ phase but decreased in the $\mathrm{G}_{2} / \mathrm{M}$ phase compared with the control (Fig. 6E). Next, the expression levels of apoptosis regulatory proteins such as Caspase 3 and PARP, were assessed. Western blotting revealed that Cleaved caspase- 3 and Cleaved PARP were increased in STK31-KD cells compared with the control (Fig. 6F). These results suggest that shRNAs targeting STK31 mRNA modulated the oncogenic potential of STK31 by inducing $\mathrm{G}_{1}$ arrest and apoptosis in $\mathrm{GC}$ cells.

STK31 overexpression promotes cell proliferation, migration and tumorigenesis. The effect of ectopic STK31 expression was assessed in MKN74 or AGS cells, in which STK31 was repressed or weakly expressed (Fig. 7A). RT-qPCR and western blotting confirmed that $S T K 31$ mRNA was stably expressed in STK31-transfected MKN74 (STK31-MKN74) and AGS (STK31-AGS) cells (Fig. 7A). Moreover, ectopic STK31 expression significantly induced colony formation in each of STK31-MKN74 and STK31-AGS cells (Fig. 7B) compared with control cells. Ectopic STK31 expression also significantly induced the proliferation (Fig. 7C) and cell migration (Fig. 7D-F) in both STK31-transfected cell lines. Finally, in vivo experiments revealed that tumors in mice engrafted with STK31-MKN74 cells were significantly larger than those of control cells (Fig. 7G and $\mathrm{H}$ ), demonstrating that tumorigenicity was promoted by ectopic $S T K 31$ expression in a xenograft mouse model. 


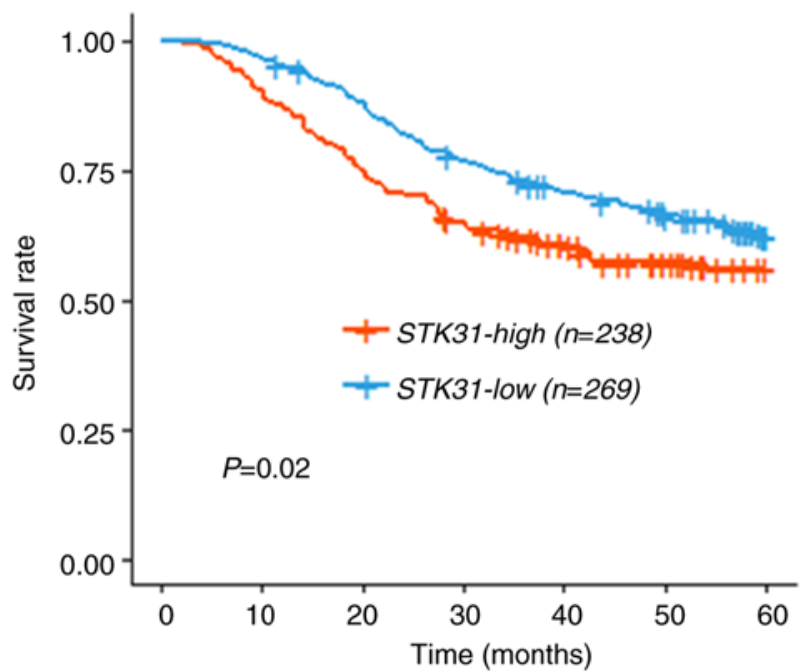

Figure 8. Survival analysis for patients with gastric cancer based on STK31 expression data. Patient data from the Chungnam National University Hospital $(n=145)$ and Samsung Medical Center $(n=432)(23)$ cohorts were merged. Data were analyzed via Kaplan-Meier method and log-rank test. STK, serine/threonine kinase.

Molecular signature of STK31 is informative regarding prognosis of patients with $G C$. In order to assess the prognostic value of $S T K 31$, clinical data from the CNUH $(n=145)$ and SMC $(n=432)$ cohorts were combined to improve predictability. Using the mean cut-off risk score, each cohort was divided into two groups based on the mean expression value of STK31, and the upper and lower groups were combined and analyzed. Kaplan-Meier survival analysis revealed a significant difference in survival rate between the two groups in the combined cohort (Fig. 8; log-rank test; $\mathrm{P}=0.02$ ), indicating that patient outcome was significantly poorer in the $S T K 31$ high expression group compared with the $S T K 31$ low expression group.

\section{Discussion}

The present results demonstrate that hypomethylation of $\mathrm{CpG}$ sites in the $S T K 31$ promoter in GC is correlated with disease progression. The present study demonstrated that DNA hypomethylation occurs in IM as well as IGC cells isolated by laser-captured microdissection, suggesting that STK31 expression may be induced during the pre-cancer IM stage. A previous study provided extensive information for $450 \mathrm{~K}$ BeadChip of gastric mucosae and IM tissue from GC-free patients, showing that $\mathrm{CpG}$ methylation at the $S T K 31$ promoter was significantly decreased in IM compared with gastric mucosae (18). TCGA Research Network has produced transcriptome and methylation data for primary GC and non-tumor tissues as a part of a study to develop a molecular classification of GC (19). Here, TCGA public data was used to show that the promoter and upstream region of $S T K 31$ were hypomethylated in primary GC and that promoter methylation was negatively correlated with STK31 mRNA expression levels. Thus, the public data regarding DNA hypomethylation at the $S T K 31$ promoter correspond well with the present results.

The STK31 promoter in GC cell lines may be key for regulating its transcription because the promoter was heavily methylated in the few GC cell lines in which STK31 was silenced. However, the association between promoter methylation and transcriptional efficiency in GC cell lines is unclear. In SNU484 cells, for example, the STK31 promoter was heavily methylated but $S T K 31$ was strongly expressed. The present data explain the association between STK31 promoter methylation and its transcription in the majority of GC cell lines, with the exception of SNU484. Further investigation is required to elucidate the association between $S T K 31$ promoter methylation and its transcription in SNU484 cells. Our results reveal that $S T K 31 \mathrm{mRNA}$ expression levels were restored in GC cell lines following treatment with 5-aza-dC and/or TSA, indicating that $S T K 31$ transcription is activated in GC cells as a consequence of drug-induced chromatin remodeling. Previously, Nano-ChIP-seq has been performed to characterize the landscapes of promoters that undergo changes in methylation in primary GC and matched normal tissue (22). Based on that data, signatures for promoter activity proximal to $S T K 31$ were analyzed, which demonstrated gain of promoter activity (increased $\mathrm{H} 3 \mathrm{~K} 4 \mathrm{me} 3$ and $\mathrm{H} 3 \mathrm{~K} 27 \mathrm{ac}$ ) at regions upstream of $S T K 31$ in primary GC but not in normal mucosae. Notably, in the present study, the regions in which promoter activity increased (chr7:23,748,702-23,749,708) overlapped partially with those in which $S T K 31$ promoter methylation decreased (chr7:23,749,662-23,749,795) in primary GC. This result suggests that the $S T K 31$ promoter may be repressed in gastric mucosae but activated in primary $\mathrm{GC}$ as a consequence of chromatin remodeling, i.e., altered DNA methylation and histone modifications.

It has been proposed that, during multistep development, human tumors acquire six hallmarks of cancer, namely sustained proliferative signaling, growth suppressor evasion, resistance to cell death, replicative immortality, induced angiogenesis and invasion and metastasis (24). Two emerging hallmarks have been added to this list, namely energy metabolism reprogramming and immune evasion (25). Genome instability and inflammation have been posited to constitute the underlying bases for these latter two hallmarks. It has been suggested that chromatin structure may be altered in response to certain hallmarks (26). The present results demonstrated that STK31 acquired aberrant gain of function in GC as a consequence of specific epigenetic alterations that promote GC cell proliferation and tumor growth both in vitro and in vivo, suggesting that STK31 serves an important role during the acquisition of certain hallmarks in numerous types of human cancer, including GC. In order to clarify the role of STK31 in vivo, however, further studies are required using a mouse model, such as tail vein injection to determine whether it causes metastasis. The downstream pathways of STK31 in the regulation of cancer cell behavior are not clear. Proteins, such as DEAD-Box helicase 4, Cullin 3 (CUL3) and Heat Shock Protein 70 superfamily, have been identified as interacting partners with STK31 in mouse testis tissue by liquid chromatography-mass spectrometry (9). CUL3 directly binds to BTB-domain containing speckle-type POZ protein (SPOP) (27). Furthermore, binding of CUL3 to SPOP, which is a candidate tumor suppressor gene in several types of cancer including GC, downregulates SPOP and thus enhances the proliferation and migration of human GC cells (28). Further investigation is required to determine whether the oncogenic potential of STK31 is achieved via interaction with CUL3 and SPOP. 
Gastric carcinogenesis proceeds through a series of precursor lesions in the GM called Correa's cascade, comprising multi-atrophic gastritis, IM, dysplasia and GC (29). In this process, IM represents a trans-differentiation of the gastric epithelium to yield an IGC, primarily induced by Helicobacter pylori infection and expression of homeobox genes, including caudal type homeobox 2 (CDX2) (30). Epidemiological evidence suggests that IM may be reversible with long-term follow up. For example, a study (31) showed that $H$. pylori eradication may reverse IM and that reversibility may be associated with a decrease in $C D X 2$ mRNA levels in patients with dysplasia as well as GC. However, the results of earlier studies on the effects of $H$. pylori eradication for improving IM have been inconsistent (32-35). Another study reported that selumetinib, an inhibitor of mitogen-activated protein kinase, may reverse IM in a mouse model based on tamoxifen injection and lead to re-establishment of normal gastric lineage (36). STK31 may be silenced in GM but activated in IM by chromatin remodeling but it is not clear whether STK31 may be a target for reversing IM in the stomach.

Taken together, the present data suggested that STK31 may be a novel IM marker that is hypomethylated longitudinally in GC and its pre-cancer lesion, IM. Furthermore, STK31 may be used as an early detection biomarker to prevent gastric carcinogenesis and predict the prognosis of patients with GC. These findings may contribute to the Pre-Cancer Atlas, a concerted initiative to characterize the molecular alterations in premalignant lesions (37). Further studies are required to clarify the exact role of $S T K 31$ in gastric carcinogenesis and to evaluate whether a small molecule or epigenetic editing could be used to modulate $S T K 31$ expression levels.

\section{Acknowledgements}

Not applicable.

\section{Funding}

The present study was funded by the National Research Foundation of Korea (grant no. 2017R1E1A1A01074883) and by the Korea Research Institute of Bioscience and Biotechnology Research Initiative.

\section{Availability of data and materials}

The data generated as a part of this study are available at the Gene Expression Omnibus (accession no. GSE55159).

\section{Authors' contributions}

YSK conceptualized and designed the study. DHB and HJK performed the experiments. YSK, DHB and HJK authenticated all the raw data. BHY and JLP operated the software. DHB and MK analyzed the data. SIL and KSS collected clinical tissue samples and pathological information. SKK and SYK interpreted data. DHB wrote the manuscript. YSK reviewed and edited the manuscript, supervised the study and obtained funding. DHB and HJK visualized the data. All authors read and approved the final version of the manuscript.

\section{Ethics approval and consent to participate}

All clinical samples were obtained with informed consent and their use was approved by the Internal Review Board at Chungnam National University Hospital (approval no. CNUH201801056006-HE001). All animal experiments were approved by the Internal Animal Care and Use Committee at Korea Research Institute of Bioscience and Biotechnology (approval no. KRIBB-AEC-16158).

\section{Patient consent for publication}

Not applicable.

\section{Competing interests}

The authors declare that they have no competing interests.

\section{References}

1. Bray F, Ferlay J, Soerjomataram I, Siegel RL, Torre LA and Jemal A: Global cancer statistics 2018: GLOBOCAN estimates of incidence and mortality worldwide for 36 cancers in 185 countries. CA Cancer J Clin 68: 394-424, 2018.

2. Orditura M, Galizia G, Sforza V, Gambardella V, Fabozzi A, Laterza MM,Andreozzi F, Ventriglia J,Savastano B, Mabilia A, et al: Treatment of gastric cancer. World J Gastroenterol 20: 1635-1649, 2014.

3. Matsuoka T and Yashiro M: Biomarkers of gastric cancer: Current topics and future perspective. World J Gastroenterol 24: 2818-2832, 2018.

4. Correa P: Human gastric carcinogenesis: A multistep and multifactorial process-First American Cancer Society Award Lecture on Cancer Epidemiology and Prevention. Cancer Res 52: 6735-6740, 1992.

5. Jones PA and Baylin SB: The fundamental role of epigenetic events in cancer. Nat Rev Genet 3: 415-428, 2002.

6. Szyf M, Pakneshan P and Rabbani SA: DNA methylation and breast cancer. Biochem Pharmacol 68: 1187-1197, 2004.

7. Upchurch GM, Haney SL and Opavsky R: Aberrant promoter hypomethylation in CLL: Does it matter for disease development? Front Oncol 6: 182, 2016.

8. Kim HJ, Kang TW, Haam K, Kim M, Kim SK, Kim SY, Lee SI Song KS, Jeong HY and Kim YS: Whole genome MBD-seq and RRBS analyses reveal that hypermethylation of gastrointestinal hormone receptors is associated with gastric carcinogenesis. Exp Mol Med 50: 1-14, 2018

9. Bao J, Wang L, Lei J, Hu Y, Liu Y, Shen H, Yan W and Xu C: STK31(TDRD8) is dynamically regulated throughout mouse spermatogenesis and interacts with MIWI protein. Histochem Cell Biol 137: 377-389, 2012.

10. Fok KL, Chen H, Ruan YC and Chan HC: Novel regulators of spermatogenesis. Semin Cell Dev Biol 29: 31-42, 2014.

11. Sabeur K, Ball BA, Corbin CJ and Conley A: Characterization of a novel, testis-specific equine serine/threonine kinase. Mol Reprod Dev 75: 867-873, 2008.

12. Xiao Y, Pollack D, Andrusier M, Levy A, Callaway M, Nieves E, Reddi $\mathrm{P}$ and Vigodner M: Identification of cell-specific targets of sumoylation during mouse spermatogenesis. Reproduction 151: 149-166, 2016.

13. Yokoe T, Tanaka F, Mimori K, Inoue H, Ohmachi T, Kusunoki M and Mori M: Efficient identification of a novel cancer/testis antigen for immunotherapy using three-step microarray analysis. Cancer Res 68: 1074-1082, 2008.

14. Kuo PL, Huang YL, Hsieh CC, Lee JC, Lin BW and Hung LY: STK31 is a cell-cycle regulated protein that contributes to the tumorigenicity of epithelial cancer cells. PLoS One 9: e93303, 2014.

15. Fok KL, Chung CM, Yi SQ, Jiang X, Sun X, Chen H, Chen YC, Kung HF, Tao Q, Diao R, et al: STK31 maintains the undifferentiated state of colon cancer cells. Carcinogenesis 33: 2044-2053, 2012.

16. Livak KJ and Schmittgen TD: Analysis of relative gene expression data using real-time quantitative PCR and the 2(-Delta Delta C(T)) method. Methods 25: 402-408, 2001. 
17. Kim M, Kim JH, Jang HR, Kim HM, Lee CW, Noh SM, Song KS, Cho JS, Jeong HY, Hahn Y, et al: LRRC3B, encoding a leucine-rich repeat-containing protein, is a putative tumor suppressor gene in gastric cancer. Cancer Res 68: 7147-7155, 2008.

18. Huang KK, Ramnarayanan K, Zhu F, Srivastava S, Xu C, Tan AL Lee M, Tay S, Das K, Xing M, et al: Genomic and epigenomic profiling of high-risk intestinal metaplasia reveals molecular determinants of progression to gastric cancer. Cancer Cell 33: 137-150.e5, 2018.

19. Cancer Genome Atlas Research Network: Comprehensive molecular characterization of gastric adenocarcinoma. Nature 513: 202-209, 2014

20. Lokk K, Modhukur V, Rajashekar B, Märtens K, Mägi R, Kolde R, Koltšina M, Nilsson TK, Vilo J, Salumets A and Tõnisson N: DNA methylome profiling of human tissues identifies global and tissue-specific methylation patterns. Genome Biol 15: r54, 2014.

21. Nazor KL, Altun G, Lynch C, Tran H, Harness JV, Slavin I, Garitaonandia I, Müller FJ, Wang YC, Boscolo FS, et al Recurrent variations in DNA methylation in human pluripotent stem cells and their differentiated derivatives. Cell Stem Cell 10: 620-634, 2012.

22. Muratani M, Deng N, Ooi WF, Lin SJ, Xing M, Xu C, Qamra A, Tay ST, Malik S, Wu J, et al: Nanoscale chromatin profiling of gastric adenocarcinoma reveals cancer-associated cryptic promoters and somatically acquired regulatory elements. Nat Commun 5: 4361, 2014.

23. Lee J, Sohn I, Do IG, Kim KM, Park SH, Park JO, Park YS, Lim HY, Sohn TS, Bae JM, et al: Nanostring-based multigene assay to predict recurrence for gastric cancer patients after surgery. PLoS One 9: e90133, 2014.

24. Hanahan D and Weinberg RA: The hallmarks of cancer. Cell 100 $57-70,2000$

25. Hanahan D and Weinberg RA: Hallmarks of cancer: The next generation. Cell 144: 646-674, 2011

26. Berdasco $M$ and Esteller M: Aberrant epigenetic landscape in cancer: How cellular identity goes awry. Dev Cell 19: 698-711, 2010

27. Zhuang M, Calabrese MF, Liu J, Waddell MB, Nourse A, Hammel M, Miller DJ, Walden H, Duda DM, Seyedin SN, et al: Structures of SPOP-substrate complexes: Insights into molecular architectures of BTB-Cul3 ubiquitin ligases. Mol Cell 36: 39-50, 2009.
28. Kim MS, Je EM, Oh JE, Yoo NJ and Lee SH: Mutational and expressional analyses of SPOP, a candidate tumor suppressor gene, in prostate, gastric and colorectal cancers. APMIS 121: 626-633, 2013

29. Correa $P$ and Piazuelo MB: The gastric precancerous cascade. J Dig Dis 13: 2-9, 2012.

30. Mesquita P, Raquel A, Nuno L, Reis CA, Silva LF, Serpa J, Van Seuningen I, Barros H and David L: Metaplasia-a transdifferentiation process that facilitates cancer development: The model of gastric intestinal metaplasia. Crit Rev Oncog 12: 3-26, 2006.

31. Shin CM, Kim N, Chang H, Kim JS, Lee DH and Jung HC: Follow-up study on CDX1 and CDX2 mRNA expression in noncancerous gastric mucosae after Helicobacter pylori eradication. Dig Dis Sci 61: 1051-1059, 2016.

32. Tucci A, Poli L, Tosetti C, Biasco G, Grigioni W, Varoli O, Mazzoni C, Paparo GF, Stanghellini V and Caletti G: Reversal of fundic atrophy after eradication of Helicobacter pylori. Am J Gastroenterol 93: 1425-1431, 1998.

33. Sung JJ, Lin SR, Ching JY, Zhou LY, To KF, Wang RT, Leung WK, Ng EK, Lau JY, Lee YT, et al: Atrophy and intestinal metaplasia one year after cure of $H$. pylori infection: A prospective, randomized study. Gastroenterology 119: 7-14, 2000.

34. Ohkusa T, Fujiki K, Takashimizu I, Kumagai J, Tanizawa T, Eishi Y, Yokoyama T and Watanabe M: Improvement in atrophic gastritis and intestinal metaplasia in patients in whom Helicobacter pylori was eradicated. Ann Intern Med 134: 380-386, 2001.

35. Kang JM, Kim N, Shin CM, Lee HS, Lee DH, Jung HC and Song IS: Predictive factors for improvement of atrophic gastritis and intestinal metaplasia after Helicobacter pylori eradication: A three-year follow-up study in Korea. Helicobacter 17: 86-95, 2012.

36. Choi E, Hendley AM, Bailey JM, Leach SD and Goldenring JR: Expression of activated ras in gastric chief cells of mice leads to the full spectrum of metaplastic lineage transitions. Gastroenterology 150: 918-930.e13, 2016.

37. Kensler TW, Spira A, Garber JE, Szabo E, Lee JJ, Dong Z, Dannenberg AJ, Hait WN, Blackburn E, Davidson NE, et al: Transforming cancer prevention through precision medicine and immune-oncology. Cancer Prev Res (Phila) 9: 2-10, 2016.

This work is licensed under a Creative Commons Attribution-NonCommercial-NoDerivatives 4.0 International (CC BY-NC-ND 4.0) License. 